\title{
Collective effects in light-heavy ion collisions
}

\author{
Björn Schenke and Raju Venugopalan \\ Physics Department, Brookhaven National Laboratory, Upton, NY 11973, USA
}

\begin{abstract}
We present results for the azimuthal anisotropy of charged hadron distributions in $\mathrm{A}+\mathrm{A}, \mathrm{p}+\mathrm{A}, \mathrm{d}+\mathrm{A}$, and ${ }^{3} \mathrm{He}+\mathrm{A}$ collisions within the IP-Glasma+Music model. Obtained anisotropies are due to the fluid dynamic response of the system to the fluctuating initial geometry of the interaction region. While the elliptic and triangular anisotropies in peripheral $\mathrm{Pb}+\mathrm{Pb}$ collisions at $\sqrt{s}=2.76 \mathrm{TeV}$ are well described by the model, the same quantities in $\sqrt{s}=5.02 \mathrm{TeV} \mathrm{p}+\mathrm{Pb}$ collisions underestimate the experimental data. This disagreement can be due to neglected initial state correlations or the lack of a detailed description of the fluctuating spatial structure of the proton, or both. We further present predictions for azimuthal anisotropies in $\mathrm{p}+\mathrm{Au}, \mathrm{d}+\mathrm{Au}$, and ${ }^{3} \mathrm{He}+\mathrm{Au}$ collisions at $\sqrt{s}=200 \mathrm{GeV}$. For $\mathrm{d}+\mathrm{Au}$ and ${ }^{3} \mathrm{He}+\mathrm{Au}$ collisions we expect the detailed substructure of the nucleon to become less important.

Keywords: heavy ion collisions, fluctuations, fluid dynamics
\end{abstract}

\section{Introduction}

For the physical interpretation of observables in heavy ion, proton/deuteron-heavy ion and (high-multiplicity) proton+proton collisions, the existence of a sophisticated description of the multi-particle production mechanism and event-by-event fluctuations is essential. In the high energy limit the color glass condensate (CGC) framework [1] is the proper effective theory of quantum chromodynamics (QCD) that provides such description.

One particular implementation of the CGC is the IP-Glasma model [2, 3]. It combines the IP-Sat dipole model $[4,5]$, which parametrizes the impact parameter and $x$-dependence of the saturation scale, with the classical dynamics of produced gluon fields $[6,7,8]$. With its parameters constrained by inclusive and diffractive deeply inelastic scattering (DIS) data from e+p scattering at HERA, the IP-Glasma model correctly describes the bulk features of various collision systems over a wide range of energies [9].

After briefly introducing the IP-Glasma model and the employed relativistic fluid dynamic simulation Music, we present results for the azimuthal anisotropy of charged hadrons produced in peripheral $\sqrt{s}=2.76 \mathrm{TeV} \mathrm{Pb}+\mathrm{Pb}$ collisions, and $\sqrt{s}=5.02 \mathrm{TeV} \mathrm{p}+\mathrm{Pb}$ collisions of comparable multiplicity. We compare to experimental data from the CMS collaboration [10]. We then present predictions for the systematics of the transverse momentum dependent anisotropy coefficients $v_{n}\left(p_{T}\right)$ in $\mathrm{p}+\mathrm{Au}, \mathrm{d}+\mathrm{Au}$, and ${ }^{3} \mathrm{He}+\mathrm{Au}$ collisions at top RHIC energies.

\section{IP-Glasma + MUSIC}

The IP-Glasma model [2, 3] relates the DIS constrained nuclear dipole cross-sections to the initial classical dynamics of highly occupied gluon fields produced in a nuclear collision. Given an initial distribution of color charges in the high energy nuclear wave-functions, the strong multiple scatterings of gluon fields are computed by event-byevent solutions of Yang-Mills equations. Both fluctuating distributions of nucleons in the nuclear wave-functions and 
intrinsic fluctuations of the color charge distributions are included. This results in "lumpy" transverse projections of the gluon field configurations that vary event to event. The scale of this lumpiness is given on average by the nuclear saturation scale $Q_{s}$ which corresponds to distance scales smaller than the nucleon size [5].

The IP-Glasma model provides the initial conditions for fluid dynamic calculations at a given time $\tau_{0}$. The initial energy density $\varepsilon$ and flow velocities $u^{\mu}$, are extracted from the gluon fields' energy-momentum tensor $T^{\mu v}$ at every transverse position via the relation $u_{\mu} T^{\mu \nu}=\varepsilon u^{v}$. In the results presented below, the viscous part of the energy momentum tensor is set to zero at the initial time of the fluid dynamic simulation. This is done because the gluon field strength tensor $T^{\mu v}$ is very anisotropic (the longitudinal pressure is approximately zero). A full 3+1 dimensional simulation including quantum fluctuations could provide a mechanism for isotropization via instabilities [11, 12].

We employ the viscous relativistic fluid dynamic simulation Music [13, 14, 15], which is a $3+1$ dimensional simulation. However, because the initial conditions from the IP-Glasma model are boost-invariant, it is used in its $2+1$ dimensional mode.

\section{3. $\mathrm{p}+\mathrm{Pb}$ collisions at the $\mathrm{LHC}$}
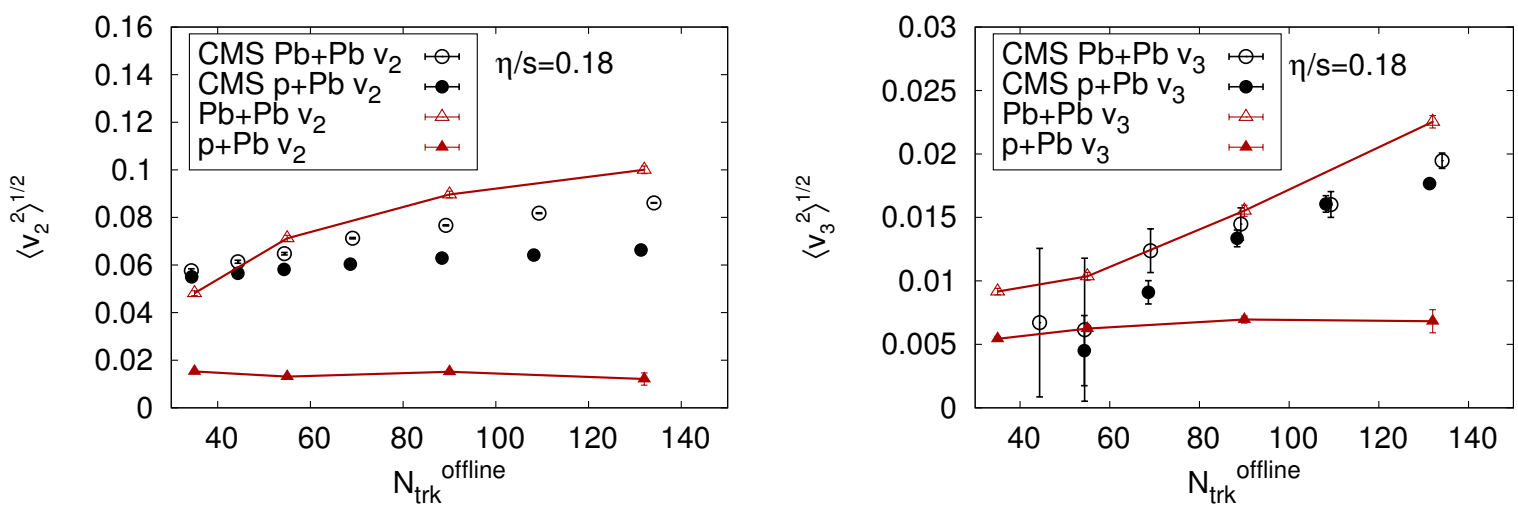

Figure 1. Multiplicity dependence of the root-mean-square elliptic (left) and triangular (right) flow coefficients in $\mathrm{Pb}+\mathrm{Pb}(\mathrm{open}$ symbols) and $\mathrm{p}+\mathrm{Pb}$ collisions (filled symbols) from the IP-Glasma+Music model (connected triangles) compared to experimental data by the CMS collaboration [10]. Figure from [16].

It was shown in [16] that experimental data from heavy ion collisions is well described by the IP-Glasma+MUSIC model out to fairly peripheral centrality bins. The natural question that arises is how the model does in describing data from $\mathrm{p}+\mathrm{A}$ collisions that produce similar multiplicities. Fig. 1 shows the multiplicity dependence of $v_{2}$ and $v_{3}$ in peripheral $\mathrm{Pb}+\mathrm{Pb}$ collisions and $\mathrm{p}+\mathrm{Pb}$ collisions with comparable multiplicity. While the agreement with experimental data from the CMS collaboration [10] is fairly good in the $\mathrm{Pb}+\mathrm{Pb}$ case, for $\mathrm{p}+\mathrm{Pb}$ collisions $v_{2}$ is under-predicted by approximately a factor of 4 . In $\mathrm{p}+\mathrm{Pb} v_{3}$ agrees for the lower multiplicities studied, but has a rather flat multiplicity dependence and underestimates the experimental data at the higher multiplicities.

One reason for the disagreement could be that all initial state correlations that lead to an elliptic anisotropy ${ }^{1}$ $[17,18,19,20,21,22,23]$ are neglected. Additionally, the description of the proton in the IP-Glasma model is oversimplified. The proton shape is approximated by a sphere, and any deviations from that are due to the small scale color charge fluctuations. Since the interaction region in $\mathrm{p}+\mathrm{Pb}$ collisions is dominated by the shape of the smaller projectile, initial geometries in $\mathrm{p}+\mathrm{Pb}$ collisions have very small eccentricities. This inevitably leads to small $v_{n}$ coefficients. If there is indeed a large contribution to $v_{n}$ coefficients in $\mathrm{p}+\mathrm{Pb}$ collisions from collective effects, our result indicates that the shape of the proton fluctuates significantly more than assumed in the IP-Glasma model. One could envision a description where the small $x$ gluon distributions are still concentrated around large $x$ valence quark positions, leading to much larger eccentricities and fluctuations. In this case $\mathrm{p}+\mathrm{A}$ collisions could be used to determine the shape and fluctuations of gluon distributions in the proton at high energies.

${ }^{1}$ When including fluctuations initial state correlations should also contribute to odd harmonics. 

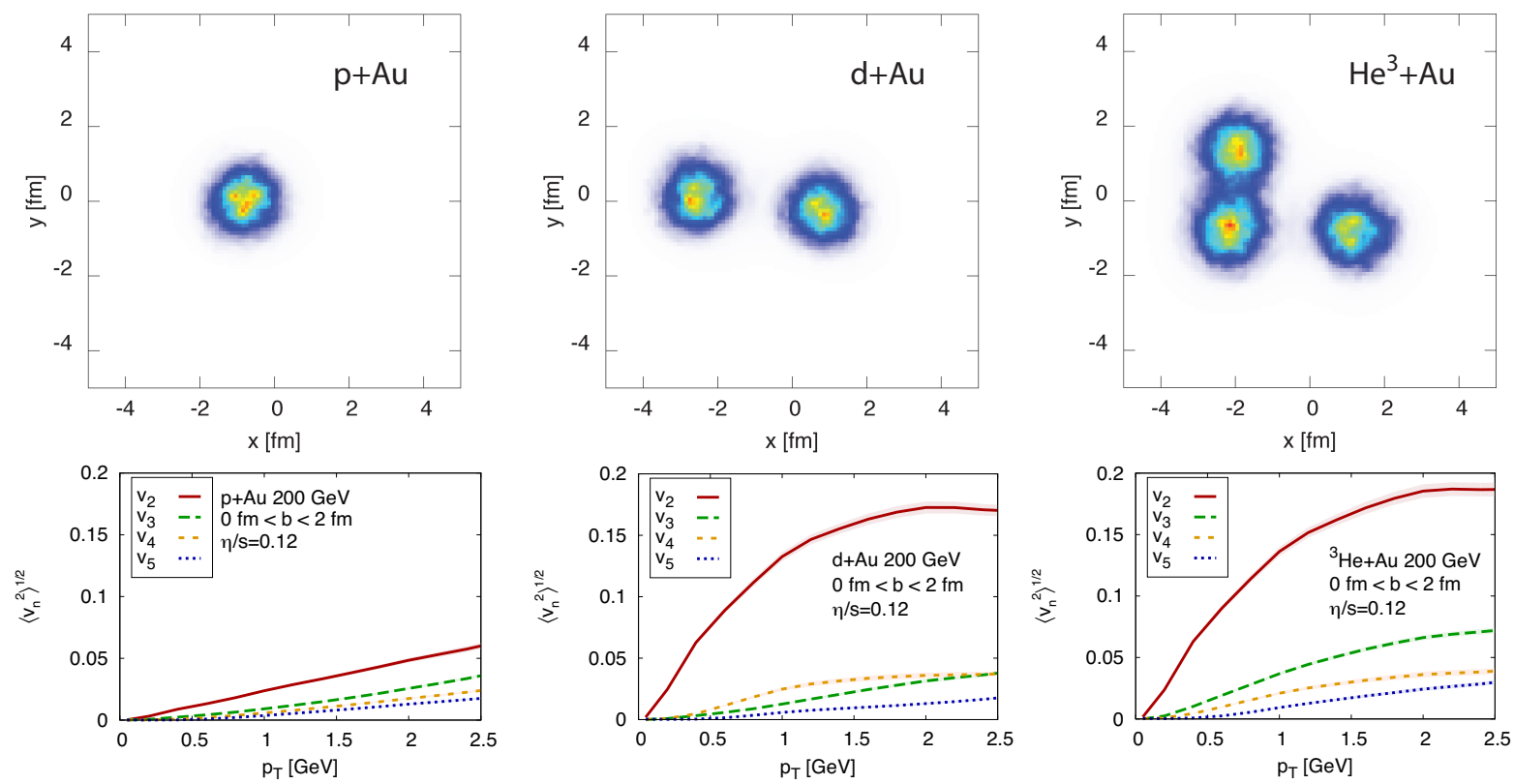

Figure 2. Typical configurations of the initial energy density distribution for $\mathrm{p}+\mathrm{Au}, \mathrm{d}+\mathrm{Au}$ and ${ }^{3} \mathrm{He}+\mathrm{Au}$ collisions (upper panel). Azimuthal anisotropy coefficients $v_{2}-v_{5}$ in the three collision systems (lower panel).

\section{4. $p+A u, d+A u$, and ${ }^{3} \mathrm{He}+A u$ collisions at $\mathrm{RHIC}$}

To determine whether final state collective effects provide the dominant contribution to the measured azimuthal anisotropy, RHIC is now studying ${ }^{3} \mathrm{He}+\mathrm{Au}$ collisions that on average generate more triangular initial state configurations compared to $\mathrm{p}+\mathrm{Au}$ or $\mathrm{d}+\mathrm{Au}$. If collectivity is the physical explanation for the observed anisotropies, we expect a larger $v_{3}$ in ${ }^{3} \mathrm{He}+\mathrm{Au}$ collisions compared to $\mathrm{p}+\mathrm{Au}$ and $\mathrm{d}+\mathrm{Au}$ collisions at the same energy. To make this expectation more quantitative, we present predictions from the IP-Glasma+Music framework.

For deuteron-gold collisions $(\mathrm{d}+\mathrm{Au})$ we compute the nucleon distribution in the deuteron using the Hulthen form of its wave function [24, 25]. For ${ }^{3} \mathrm{He}$, we use the same nucleon configurations as employed in [26]. They are obtained from Green's function Monte Carlo calculations using the AV18 + UIX model interaction [27].

For this comparative study we do not perform a detailed centrality selection, but instead sample the impact parameter $b$ between 0 and $2 \mathrm{fm}$ in all systems. We then compute the initial state distribution of the energy density and flow velocity at time $\tau_{0}=0.5 \mathrm{fm} / c$ and evolve the system using viscous fluid dynamics with $\eta / s=0.12$ until freeze-out at $T=135 \mathrm{MeV}$.

We present typical configurations of the initial energy density distribution in the transverse plane and final results for the transverse momentum dependent azimuthal anisotropy coefficients $v_{2}$ to $v_{5}$ in Fig. 2 . While we find very small values for $v_{2}$ through $v_{5}$ in $\mathrm{p}+\mathrm{Au}$ collisions, the additional nucleons and their position fluctuations generate larger $v_{2}-v_{4}$ in $\mathrm{d}+\mathrm{Au}$ and ${ }^{3} \mathrm{He}+\mathrm{Au}$ collisions. The odd harmonics $v_{3}$ and $v_{5}$ are noticeably larger in ${ }^{3} \mathrm{He}+\mathrm{Au}$ collisions compared to $\mathrm{d}+\mathrm{Au}$ collisions. This qualitative prediction can be compared to future measurements at RHIC.

\section{Conclusions}

We have demonstrated that experimental results for $v_{2}$ and $v_{3}$ in proton-heavy ion collisions at the LHC are not well described by the IP-Glasma+Music model. Reasons for this could be the neglected initial state correlations and/or the lack of a detailed description of the fluctuating subnucleonic structure of the proton. Our results for $\mathrm{p}+\mathrm{A}$ collisions differ significantly from those in $[28,29,30,31]$, suggesting that the details of the initial shape in small systems are of paramount importance. 
We predict an increase of both $v_{3}$ and $v_{5}$ in ${ }^{3} \mathrm{He}+\mathrm{Au}$ collisions compared to $\mathrm{d}+\mathrm{Au}$ collisions, while the even harmonics are comparable in both systems. The detailed substructure of the nucleon is expected to be less important for the initial state geometry in these collisions compared to $\mathrm{p}+\mathrm{A}$ collisions.

Acknowledgments We thank Jamie Nagle for providing us with the ${ }^{3} \mathrm{He}$ nucleon configurations. This work was supported under DOE Contract No. DE-AC02-98CH10886. BPS acknowledges support from a DOE Office of Science Early Career Award. This research used resources of the National Energy Research Scientific Computing Center, which is supported by the DOE Office of Science under Contract No. DE-AC02-05CH11231.

\section{References}

[1] F. Gelis, E. Iancu, J. Jalilian-Marian, R. Venugopalan, The Color Glass Condensate, Ann.Rev.Nucl.Part.Sci. 60 (2010) $463-489$.

[2] B. Schenke, P. Tribedy, R. Venugopalan, Fluctuating Glasma initial conditions and flow in heavy ion collisions, Phys. Rev. Lett. 108 (2012) 252301.

[3] B. Schenke, P. Tribedy, R. Venugopalan, Event-by-event gluon multiplicity, energy density and eccentricities at RHIC and LHC, Phys. Rev. C86 (2012) 034908

[4] H. Kowalski, D. Teaney, An impact parameter dipole saturation model, Phys. Rev. D68 (2003) 114005.

[5] H. Kowalski, T. Lappi, R. Venugopalan, Nuclear enhancement of universal dynamics of high parton densities, Phys. Rev. Lett. 100 (2008) 022303.

[6] A. Krasnitz, R. Venugopalan, The initial energy density of gluons produced in very high energy nuclear collisions, Phys. Rev. Lett. 84 (2000) 4309-4312.

[7] A. Krasnitz, R. Venugopalan, The initial gluon multiplicity in heavy ion collisions, Phys. Rev. Lett. 86 (2001) 1717-1720.

[8] T. Lappi, Production of gluons in the classical field model for heavy ion collisions, Phys. Rev. C67 (2003) 054903.

[9] B. Schenke, P. Tribedy, R. Venugopalan, Multiplicity distributions in p+p, p+A and A+A collisions from Yang-Mills dynamics, Phys.Rev. C89 (2014) 024901.

[10] S. Chatrchyan, et al., Multiplicity and transverse momentum dependence of two- and four-particle correlations in $\mathrm{pPb}$ and $\mathrm{PbPb}$ collisions, Phys.Lett. B724 (2013) 213-240.

[11] J. Berges, K. Boguslavski, S. Schlichting, R. Venugopalan, Turbulent thermalization process in heavy-ion collisions at ultrarelativistic energies, Phys.Rev. D89 (2014) 074011

[12] T. Epelbaum, F. Gelis, Pressure isotropization in high energy heavy ion collisions, Phys.Rev.Lett. 111 (2013) 232301.

[13] B. Schenke, S. Jeon, C. Gale, (3+1)D hydrodynamic simulation of relativistic heavy-ion collisions, Phys. Rev. C82 (2010) 014903.

[14] B. Schenke, S. Jeon, C. Gale, Elliptic and triangular flow in event-by-event (3+1)D viscous hydrodynamics, Phys. Rev. Lett. 106 (2011) 042301.

[15] B. Schenke, S. Jeon, C. Gale, Higher flow harmonics from (3+1)D event-by-event viscous hydrodynamics, Phys. Rev. C85 (2011) 024901.

[16] B. Schenke, R. Venugopalan, Eccentric protons? Sensitivity of flow to system size and shape in $\mathrm{p}+\mathrm{p}, \mathrm{p}+\mathrm{Pb}$ and $\mathrm{Pb}+\mathrm{Pb}$ collisions , arXiv: 1405.3605

[17] K. Dusling, R. Venugopalan, Azimuthal collimation of long range rapidity correlations by strong color fields in high multiplicity hadronhadron collisions, Phys.Rev.Lett. 108 (2012) 262001.

[18] K. Dusling, R. Venugopalan, Evidence for BFKL and saturation dynamics from dihadron spectra at the LHC, Phys.Rev. D87 (5) (2013) 051502.

[19] K. Dusling, R. Venugopalan, Explanation of systematics of CMS p+Pb high multiplicity di-hadron data at $\sqrt{s}_{\mathrm{NN}}=5.02 \mathrm{TeV}$, Phys.Rev. D87 (5) (2013) 054014.

[20] K. Dusling, R. Venugopalan, Comparison of the Color Glass Condensate to di-hadron correlations in proton-proton and proton-nucleus collisions, Phys.Rev. D87 (2013) 094034.

[21] M. Gyulassy, P. Levai, I. Vitev, T. Biro, Non-Abelian Bremsstrahlung and Azimuthal Asymmetries in High Energy p+A Reactions , arXiv: 1405.7825.

[22] A. Dumitru, A. V. Giannini, Initial state angular asymmetries in high energy p+A collisions: spontaneous breaking of rotational symmetry by a color electric field and C-odd fluctuations , arXiv:1406.5781.

[23] L. McLerran, V. V. Skokov, The Eccentric Collective BFKL Pomeron, arXiv:1407.2651.

[24] M. L. Miller, K. Reygers, S. J. Sanders, P. Steinberg, Glauber modeling in high energy nuclear collisions, Ann. Rev. Nucl. Part. Sci. 57 (2007) $205-243$.

[25] A. Bzdak, B. Schenke, P. Tribedy, R. Venugopalan, Initial state geometry and the role of hydrodynamics in proton-proton, proton-nucleus and deuteron-nucleus collisions, Phys.Rev. C87 (6) (2013) 064906.

[26] J. Nagle, A. Adare, S. Beckman, T. Koblesky, J. O. Koop, et al., Exploiting Intrinsic Triangular Geometry in Relativistic He3+Au Collisions to Disentangle Medium Properties, arXiv:1312.4565.

[27] J. Carlson, R. Schiavilla, Structure and dynamics of few nucleon systems, Rev.Mod.Phys. 70 (1998) $743-842$

[28] P. Bozek, W. Broniowski, Correlations from hydrodynamic flow in p-Pb collisions, Phys.Lett. B718 (2013) 1557-1561, doi: 10.1016/j.physletb.2012.12.051.

[29] K. Werner, M. Bleicher, B. Guiot, I. Karpenko, T. Pierog, Evidence for flow in pPb collisions at 5 TeV from v2 mass splitting, Phys.Rev.Lett. 112 (2014) 232301, doi:10.1103/PhysRevLett.112.232301.

[30] I. Kozlov, M. Luzum, G. Denicol, S. Jeon, C. Gale, Transverse momentum structure of pair correlations as a signature of collective behavior in small collision systems, arXiv:1405.3976.

[31] A. Bzdak, G.-L. Ma, Elliptic and triangular flow in $\mathrm{p}+\mathrm{Pb}$ and peripheral $\mathrm{Pb}+\mathrm{Pb}$ collisions from parton scatterings , arXiv:1406.2804. 